\title{
HOMOTOPY CLASSIFICATION OF ELLIPTIC PROBLEMS ASSOCIATED WITH DISCRETE GROUP ACTIONS ON MANIFOLDS WITH BOUNDARY
}

\author{
A.YU. SAVIN, B.YU. STERNIN
}

\begin{abstract}
Given an action of a discrete group $G$ on a smooth compact manifold $M$ with a boundary, we consider a class of operators generated by pseudodifferential operators on $M$ and shift operators associated with the group action. For elliptic operators in this class, we obtain a classification up to stable homotopies and show that the group of stable homotopy classes of such problems is isomorphic to the $K$-group of the crossed product of the algebra of continuous functions on the cotangent bundle over the interior of the manifold and the group $G$ acting on this algebra by automorphisms.
\end{abstract}

Keywords: elliptic operator, homotopy classification, $K$-theory, crossed product, $G$ operator.

Mathematics subject classification (2010): Primary 58J32; Secondary 58J40, 46L80, $35 \mathrm{~S} 15$

\section{INTRODUCTION}

The index problem in elliptic theory is to express the index of an elliptic operator in terms of the topological invariants of its symbol and the manifold, on which this operator is defined 1. Here a description of the homotopy classification of operators plays an important role; this classification means to calculate the group of (stable) homotopy classes of elliptic operators on the manifold. The benefit of such calculation is that the homotopy invariants of the operators, and, in particular, the index, are functionals on this group of the homotopy classes.

The homotopy classification was obtained first on a smooth closed manifold in [1], where it was shown that the group of stably homotopy classes is isomorphic to a topological $K$-group with compact supports $K_{c}\left(T^{*} M\right)$ of the cotangent bundle of the manifold. Then the homotopy classification was obtained for many other interesting elliptic operators. For instance, it was shown in [2] that the homotopy classification of classical boundary value problems on a manifold with a boundary can be obtained in terms of the group $K_{c}\left(T^{*} M^{\circ}\right)$ associated with the interior $M^{\circ}=M \backslash \partial M$ of the manifold with a boundary. The homotopy classification of elliptic operators in Boutet de Monvel's algebra was obtained on a manifold with a boundary [3, 4]. The homotopy classification was also obtained for many classes of manifolds with singularities [5]-[7]. There were considered the applications of the classification to calculating obstruction of Atiyah-Bott type [8] for the existence of elliptic problems on manifolds with singularities, to the description of Poincaré duality on manifolds with singularities and others [9]-[13].

A.Yu. Savin, B.Yu Sternin, Homotopy classification of elliptic problems associated with DISCRETE GROUP ACTIONS ON MANIFOLDS WITH BOUNDARY.

(c)SAVin A.Yu., Sternin B.Yu. 2016.

The work is partially supported by the RFBR grants (nos. 15-01-08392 and 16-01-00373), by the Ministry of Education and Science of Russia (agreement no. 02.a03.21.0008), and by a grant of Deutsche Forschungsgemeinschaft.

Submitted May 18, 2016. 
There is an interesting class of elliptic problems associated with the groups actions on manifolds (see the monographs and survey [14]-[17] and the references therein). For these problems on a smooth closed manifold there was obtained a homotopy classification in terms of $K$-group $K_{0}\left(C_{0}\left(T^{*} M\right) \rtimes G\right)$ of the crossed product of the algebra of continuous functions on the cotangent bundle $T^{*} M$ and by the group $G$ acting by the automorphisms on this algebra. The latter $K$-group can be calculated in the topological terms for many groups $G$ thanks to the Baum-Connes mapping (isomorphism) [18].

The aim of the present work is to obtain the homotopy classfication of elliptic pseudodifferential operators associated with the action of a discrete group on a manifold with a boundary. We note that the theory of general pseudodifferential operators on a manifold with a boundary was described in works [19]-21]. In the present work we lean on the description of the corresponding $C^{*}$-algrebra of such operators given in work [22]. We show that the group of stably homotopy classes is isomorphic to the $K$-group $K_{0}\left(C_{0}\left(T^{*} M^{\circ}\right) \rtimes G\right)$ of the crossed product associated with the interior of the manifold.

\section{Formulation of the Problem}

2.1. Pseudodifferential operators on a manifold with a boundary. In this subsection we recall main facts on the structure of the algebra of pseudodifferential operators on a manifold with a boundary from work [22]. Let $M$ be a smooth compact manifold with a boundary $X=\partial M$. We assume that some collar neighbourhood $U$ of the boundary is chosen, that is, a diffeomorphism

$$
U \simeq X \times[0,1)
$$

under which the boundary $X$ is mapped into the manifold $X \times\{0\}$. In what follows, as local coordinates in a neighbourhood of the boundary we choose $(y, t)$, where $y$ are coordinates on $X$, and $t \in[0,1)$.

By $\Psi(M) \subset \mathcal{B} L^{2}(M)$ we denote the $C^{*}$-algebra of pseudodifferential operators of zero order on $M$, generally speaking, without transmission property [22] and acting in space $L^{2}$ on the manifolds. The Calkin's algebra is denoted by $\Sigma=\Psi(M) / \mathcal{K}$. Hereinafter by $\mathcal{K}$ we denote the ideal of compact operators. Let us recall the needed to us facts on the algebra $\Sigma$ established in the cited work. There exists a symbolic mapping

$$
\sigma=\left(\sigma_{\text {int }}, \sigma_{b}\right): \Sigma \longrightarrow C\left(S^{*} M\right) \oplus C\left(S^{*} X, \mathcal{B} L^{2}\left(\mathbb{R}_{+}\right)\right),
$$

whose components are called interior and boundary symbol, respectively. The $C^{*}$-algebra of boundary symbols denote by

$$
\Sigma_{b}=\operatorname{Im} \sigma_{b} \subset C\left(S^{*} X, \mathcal{B} L^{2}\left(\mathbb{R}_{+}\right)\right),
$$

has the following additional symbolic structure: on this algebra, the mapping of the interior symbol is defined

and of Mellin symbol

$$
\sigma_{i n t}^{\prime}: \Sigma_{b} \longrightarrow C\left(\left.S^{*} M\right|_{X}\right)
$$

$$
\sigma_{M}: \Sigma_{b} \longrightarrow C(X \times \overline{\mathbb{R}})
$$

the exact sequence of $C^{*}$-algebras

$$
0 \longrightarrow C\left(S^{*} X, \mathcal{M}_{0}\right) \longrightarrow \Sigma_{b} \stackrel{\sigma_{M}}{\longrightarrow} C(X \times \overline{\mathbb{R}}) \longrightarrow 0
$$

holds, where $\mathcal{M}_{0} \subset \mathcal{B} L^{2}\left(\mathbb{R}_{+}\right)$is the ideal formed by the boundary symbols with zero Mellin symbol. We note that the boundary symbols corresponding to this ideal satisfy the transmission condition, while this ideal coincides with the ideal considered in Theorem 2 in [3]. In particular, it was established in the cited work that this ideal has trivial $K$-groups

$$
K_{*}\left(\mathcal{M}_{0}\right)=0 .
$$


In the local coordinates $(y, t)$ in a neighbourhood of the boundary the boundary symbol can be obtained from the operator by freezing its coefficients at a point of the boundary and applying then Fourier transform $y \rightarrow \eta$. We obtain an operator in the space with the coordinates $\eta, t$, which is a family of operators acting in the space of $L^{2}$-functions on the half-line $\mathbb{R}_{+}$with the coordinate $t$ and depending on the parameters $\eta$. This family is exactly the boundary symbol.

The Mellin symbol $\sigma_{M}(a)$ of a boundary symbol $a \in \Sigma_{b}$ is introduced as follows. The boundary symbol $a$ is a family of operators on the half-line $\overline{\mathbb{R}}_{+}$. Then the zero $0 \in \overline{\mathbb{R}}_{+}$ is considered as a conical point and the Mellin symbol is just the conormal symbol of the considered operator at this point. In other words, we freeze the coefficients of the operator $a$ at zero and make the Mellin transform $t \rightarrow p$. The boundary symbol is mapped in the operator of multiplication by a function of the variable $p$, which, by the definition, is the Mellin symbol of the operator.

2.2. $G$-pseudodifferential operators on a manifold with a boundary. We assume in addition that the action by diffeomorphisms of a discrete group $G$ on $M$ is given. We also assume that this group is amenable [23. A class of operators called $G$-operators is associated with the action of a group on a manifold [17, 24]. Let us define these operators in the considered situation.

The group $G$ acts on the manifold and therefore, it acts by means of the corresponding change of variables by the automorphisms on the $C^{*}$-algebra $C(M)$ of continuous functions on the manifold $M$, as well as on the algebra $\Psi(M)$ of pseudodifferential operators. The $C^{*}$-crossed product

$$
\Psi(M) \rtimes G
$$

is associated with the action of the algebra $\Psi(M)[16],[24]$. By the amenability of the group the crossed product is defined uniquely. The elements of this $C^{*}$-algebra are families $\left\{D_{g}\right\}_{g \in G}$ of pseudodifferential operators $D_{g} \in \Psi(M)$ parameterized by the group $G$. To this family, we associated a so-called $G$-operator

$$
D=\sum_{g \in G} D_{g} U_{g}: L^{2}(M) \longrightarrow L^{2}(M),
$$

where

$$
U_{g}: u(x) \longmapsto\left(\frac{\left(g^{-1}\right)^{*} \mu}{\mu}(x)\right)^{1 / 2} u\left(g^{-1} x\right)
$$

is the unitary representation of the group by means of the weighted shift operators in the space $L^{2}(M)$. Here $\mu$ is the volume form on $M$ defining the scalar product in the space $L^{2}(M)$. The sum in (4) is well-defined for compactly supported in $g$ families and can be extended to the entire crossed product by the universal property of the crossed products (for details see [17, 24]) and thus, the representation

$$
\begin{array}{ccc}
\Psi(M) \rtimes G & \longrightarrow & \mathcal{B} L^{2}(M) \\
\left\{D_{g}\right\} & \longmapsto & D=\sum_{g \in G} D_{g} U_{g}
\end{array}
$$

of the crossed product is well-defined in the space $L^{2}(M)$. For the obtained operator we introduce the notion of the symbol

$$
\sigma(D)=\left\{\sigma\left(D_{g}\right)\right\}_{g \in G} \in \Sigma \rtimes G,
$$

the ellipticity condition is formulated as the invertibility condition of the symbol in the mentioned algebra (in the cited works more explicit descriptions of this condition are also given) and it is shown that the elliptic operators are Fredholm.

Apart from scalar operators (4), we can also consider the corresponding matrix operator. However, we note that it is more natural to make the homotopy classification in terms of a wider class of operators than operators (4) or even matrix operators being analogues of operators 
acting in the sections of the vector bundles (see [1] in the classical case). Namely, we consider the class of the operators of the form

$$
D: \operatorname{Im} P_{1} \longrightarrow \operatorname{Im} P_{2}, \quad \operatorname{Im} P_{1,2} \subset L^{2}\left(M, \mathbb{C}^{N}\right),
$$

acting between the ranges of the matrix projectors

$$
P_{1,2} \in \operatorname{Mat}_{N}(C(M) \rtimes G)
$$

(that is, the relations $\left(P_{1}\right)^{2}=P_{1},\left(P_{2}\right)^{2}=P_{2}$ hold true) with the entries in the algebra $C(M) \rtimes G$, and

$$
D \in \operatorname{Mat}_{N}(\Psi(M) \rtimes G)
$$

is a matrix operator with the entries in the crossed product $\Psi(M) \rtimes G$. The projectors $P_{1}, P_{2}$ and operators $D$ satisfy the relation

$$
P_{2} D P_{1}=D P_{1}
$$

which means that the operator $D$ maps the range of the projector $P_{1}$ into the range of the projector $P_{2}$. For the operators of form (5) we naturally introduce the notion of the symbol and the ellipticity and the Fredholm property theorem is true (see the abstract construction in [5]).

2.3. Problem on homotopy classification. By $\operatorname{Ell}(M, G)$ we denote the Abelian group of stably homotopy classes of elliptic operators of form (5). We recall briefly (for details see [5]) that two operators of such type are called stably homotopy if there exists a continuous homotopy of elliptic operators $\left(D_{t}, P_{1, t}, P_{2, t}\right)$ connecting direct sums of these operators with some trivial operators. Here, as a trivial operator, we call operators of form (5) in which the operator $D$ has the entries in the subalgebra

$$
C(M) \rtimes G \subset \Psi(M) \rtimes G .
$$

In the standard way one can check that the stable homotopy is an equivalence relation on the set of elliptic operators of form (5).

The aim of the work is to obtain a stably homotopy classification, that is, to calculate the group $\operatorname{Ell}(M, G)$ in terms of the topological invariants of the action of the group on the manifold.

\section{MAIN RESUlT}

The main result of this work is given in a theorem below. In order to state it, we need the definition of an admissible action.

Definition 1. The action of a group $G$ on a manifold $M$ with a boundary is called admissible if one of the following two conditions is satisfied:

1) either for each element $g \in G$, the induced action on the cotangent bundle by means of the differentials

$$
\partial g=\left(d g^{t}\right)^{-1}: T^{*} M \longrightarrow T^{*} M, \text { where } d g: T M \rightarrow T M \text { is a differential, }
$$

over the boundary (i.e., as $t=0$ ) is equal to

$$
\left.\partial g\right|_{t=0}=\left(\begin{array}{cc}
\partial\left(\left.g\right|_{X}\right) & 0 \\
0 & i d
\end{array}\right): T^{*} X \oplus \mathbb{R} \longrightarrow T^{*} X \oplus \mathbb{R}
$$

where we employ the expansion

$$
\left.T^{*} M\right|_{X} \simeq T^{*} X \oplus \mathbb{R}
$$

corresponding to collar neighbourhood (1). In other words, as $t=0$, the co-differential acts identically along the normal direction to the boundary, while along the directions tangent to the boundary it coincides with the differential of the restriction of the action on the boundary; 
2) or for an arbitrary $C^{*}$-algebra $A$, on which the group $G$ acts, the triviality of its $K$-groups $K_{*}(A)=0$ implies the same for the $K$-group of the crossed product $K_{*}(A \rtimes G)=0$.

We observe that the class of admissible actions includes arbitrary actions of finite groups or, more general, an arbitrary isometric action (in this Condition 1) is satisfied), an arbitrary action of the group $\mathbb{Z}^{n}$ for $n$ (here Condition 2) is satisfied), one can easily confirm this by employing Pimsner-Voiculescu sequence [25].

Theorem 1. Assume that the action of a group $G$ on the manifold $M$ is admissible. Then the isomorphism of the groups

$$
\operatorname{Ell}(M, G) \simeq K_{0}\left(C_{0}\left(T^{*} M^{\circ}\right) \rtimes G\right)
$$

holds true, where $M^{\circ}=M \backslash X$ is the interior of the manifold. Here, on the cotangent bundle $T^{*} M^{\circ}$ we consider the following action of the group $G$ :

$$
(x, \xi) \in T^{*} M \longmapsto\left(g x,|\xi| \frac{\partial g \xi}{|\partial g \xi|}\right) \in T^{*} M,
$$

where the norms of the covectors are calculated with respect to some fixed metric on the manifold.

Isomorphism (7) can be treated as follows (cf. [8, 2, 3]): the group $\operatorname{Ell}(M, G)$ is isomorphic to an analogous group for some narrower (and simpler) class of operators, which are isomorphisms over the boundary and an arbitrary operator is stably homotopy to an operator in this class.

Remark 1. For the trivial group $G=\{e\}$ this theorem gives the isomorphism

$$
\operatorname{Ell}(M,\{e\}) \simeq K_{0}\left(C_{0}\left(T^{*} M^{\circ}\right)\right) \simeq K_{c}\left(T^{*} M^{\circ}\right),
$$

that is in agreement with the results on the classification of classical boundary value problem and pseudodifferential operators with the transmission property [2]-[4].

Remark 2. For many discrete groups, the K-group of the crossed product in (7) can be calculated in topological terms by employing Baum-Connes mapping with coefficients (see [18]). For instance,

1) for a finite group $G$ we obtain the isomorphism

$$
K_{0}\left(C_{0}\left(T^{*} M^{\circ}\right) \rtimes G\right) \simeq K_{0}^{G}(M)
$$

with the even group of $G$-equivalent $K$-homologies of the manifold $M$;

2) for the group $G=\mathbb{Z}^{n}$ we have

$$
K_{0}\left(C_{0}\left(T^{*} M^{\circ}\right) \rtimes \mathbb{Z}^{n}\right) \simeq K_{0}\left(M \times \mathbb{R}^{n} / \mathbb{Z}^{n}\right),
$$

where in the right hand side we have the K-homology group of the quotient space of the product $M \times \mathbb{R}^{n}$ with respect to the diagonal action of the group $\mathbb{Z}^{n}$ (the diagonal ation is free and proper and this is why the factor space is a smooth manifold).

Remark 3. In the case, when the action of the group is isometric, Theorem 1 can be applied for proving the index formula. In order to do it, we need just to construct the topological index by using the methods in [16, 26]. These issues are planned to be considered in other work.

\section{PROOF OF THE MAIN THEOREM}

Here we prove Theorem 1 .

1. First we express the group $\operatorname{Ell}(M, G)$ of stable homotopy classes of elliptic operators in terms of the $K$-group of some $C^{*}$-algebra associated with the algebra of the symbols. Namely, by the results of work [5] we have the isomorphism of Abelian groups

$$
\operatorname{Ell}(M, G) \simeq K_{0}(\operatorname{Con}(C(M) \rtimes G \rightarrow \Sigma \rtimes G))=K_{0}(\operatorname{Con}(C(M) \rightarrow \Sigma) \rtimes G),
$$


where for a homomorphism $f: A \rightarrow B$ of $C^{*}$-algebras $A$ and $B$, by

$$
\operatorname{Con}(A \rightarrow B)=\{(a, b(t)) \in A \oplus C[0,1) \otimes B \mid f(a)=b(0)\}
$$

we denote the cone of this homomorphism.

2. We consider the ideals

$$
C_{0}\left(M^{\circ}\right) \subset C(M), \quad \Sigma_{0}=C_{0}\left(S^{*} M^{\circ}\right) \subset \Sigma
$$

consisting of the functions and symbols vanishing at the boundary. The ideals in (9) give us a short exact sequence

$$
0 \rightarrow \operatorname{Con}\left(C_{0}\left(M^{\circ}\right) \rightarrow \Sigma_{0}\right) \rtimes G \stackrel{i}{\rightarrow} \operatorname{Con}(C(M) \rightarrow \Sigma) \rtimes G \rightarrow \operatorname{Con}\left(C(X) \rightarrow \Sigma_{b}\right) \rtimes G
$$

of the crossed products of the cones for the corresponding mapping, where $\Sigma_{b} \subset$ $C\left(S^{*} X, \mathcal{B} L^{2}\left(\mathbb{R}_{+}\right)\right)$stands for the algebra of the boundary symbols. The exact sequence in $K$-theory

$$
\begin{gathered}
\ldots \longrightarrow K_{*+1}\left(\operatorname{Con}\left(C(X) \rightarrow \Sigma_{b}\right) \rtimes G\right) \stackrel{\partial}{\longrightarrow} K_{*}\left(\operatorname{Con}\left(C_{0}\left(M^{\circ}\right) \rightarrow \Sigma_{0}\right) \rtimes G\right) \stackrel{i_{*}}{\longrightarrow} \\
\stackrel{i_{*}}{\longrightarrow} K_{*}(\operatorname{Con}(C(M) \rightarrow \Sigma) \rtimes G) \longrightarrow K_{*}\left(\operatorname{Con}\left(C(X) \rightarrow \Sigma_{b}\right) \rtimes G\right) \rightarrow \ldots
\end{gathered}
$$

corresponding to short sequence $(10)$ implies that the embedding $i$ induces the isomorphism of $K$-groups if $K$-groups of the cone $\operatorname{Con}\left(C(X) \rightarrow \Sigma_{b}\right) \rtimes G$ are trivial.

Now we assume that the action of the group is admissible in the sense of Definition 1 and Condition 1) holds true. The case, when Condition 2) holds true, will be considered later. Then we have the isomorphism of $C^{*}$-algebras

$$
\operatorname{Con}\left(C_{0}\left(M^{\circ}\right) \rightarrow \Sigma_{0}\right) \rtimes G \simeq C_{0}\left(T^{*} M^{\circ}\right) \rtimes G
$$

and therefore, we have the isomorphism of their $K$-groups. This isomorphism and the isomorphism $i_{*}$ in (11) lead us to the statement of the theorem.

Thus, in order to prove the theorem, it is sufficient to establish the triviality of the $K$-groups of the cone

$$
\operatorname{Con}\left(C(X) \rightarrow \Sigma_{b}\right) \rtimes G .
$$

By the exact sequence for the cone in $K$-theory (see, for instance, [27]), cone (13) has trivial $K$-groups if the embedding

$$
C(X) \rtimes G \longrightarrow \Sigma_{b} \rtimes G,
$$

to which it is associated, induces the isomorphism in $K$-theory.

3. The mapping of the Mellin symbol

$$
\sigma_{M}: \Sigma_{b} \rightarrow C(X \times \overline{\mathbb{R}})
$$

gives a short exact sequence of $C^{*}$-algebras

$$
0 \rightarrow\left(C\left(S^{*} X\right) \otimes \mathcal{M}_{0}\right) \rtimes G \longrightarrow \Sigma_{b} \rtimes G \stackrel{\sigma_{M}}{\longrightarrow} C(X \times \overline{\mathbb{R}}) \rtimes G \longrightarrow 0,
$$

where $\mathcal{M}_{0} \subset \mathcal{B} L^{2}\left(\mathbb{R}_{+}\right)$is the ideal consisting of boundary symbols with zero Mellin symbol. Here we observe the isomorphism of $C^{*}$-algebras

$$
\left(C\left(S^{*} X\right) \otimes \mathcal{M}_{0}\right) \rtimes G \simeq\left(C\left(S^{*} X\right) \rtimes G\right) \otimes \mathcal{M}_{0}
$$

implied by the fact that by our assumption the group $G$ acts trivially with respect the variable $t$, that is, it gives the identity mapping on the algebra $\mathcal{M}_{0}$. Then the algebra $\mathcal{M}_{0}$ coincides with the ideal considered in work [3, where it was established that this ideal has trivial $K$ groups. By Künneth formula we also obtain that algebra (16) has trivial $K$-groups and by the exactness of the sequence in $K$-theory for pair (15) we obtain that the Mellin symbol induces an isomorphism of $K$-groups

$$
\sigma_{M *}: K_{*}\left(\Sigma_{b} \rtimes G\right) \longrightarrow K_{*}(C(X \times \overline{\mathbb{R}}) \rtimes G) \simeq K_{*}(C(X) \rtimes G) .
$$


Then it is obvious that embedding (14) defines the right inverse mapping for the mapping $\sigma_{M}$. And since $\sigma_{M *}$ is an isomorphism in $K$-theory, the mentioned embedding defines also an isomorhism in $K$-theory.

4. Thus, we have established that isomorphism (14) induces the isomorphism of $K$-groups and therefore, its cone (13) has trivial $K$-groups. This is why the embedding $i$ in $(10)$ induces an isomoprhism of $K$-groups and in view of (8) and $(12)$ we finally obtain:

$\operatorname{Ell}(M, G) \simeq K_{0}(\operatorname{Con}(C(M) \rightarrow \Sigma) \rtimes G) \stackrel{i_{*}^{-1}}{\simeq} K_{0}\left(\operatorname{Con}\left(C_{0}\left(M^{\circ}\right) \rightarrow \Sigma_{0}\right) \rtimes G\right) \simeq K_{0}\left(C_{0}\left(T^{*} M^{\circ}\right) \rtimes G\right)$.

Theorem 1 has been proved for the case, when admissibility condition 1) is satisfied.

5. Let us prove the theorem for the actions satisfying the admissibility condition 2). For such actions the proof follows the same lines as above except the only difference: formula (16) is no longer true since the action of the group is not identical on the algebra $\mathcal{M}_{0}$. However, by the Künneth formula we have

$$
K_{*}\left(C\left(S^{*} X\right) \otimes \mathcal{M}_{0}\right)=0
$$

that by the above condition for the group yields the triviality of $K$-groups of the crossed products

$$
K_{*}\left(\left(C\left(S^{*} X\right) \otimes \mathcal{M}_{0}\right) \rtimes G\right)=0 .
$$

Other parts of the proof are reproduced with no changes.

The proof of Theorem 1 is complete.

\section{BIBLIOGRAPHY}

1. M. F. Atiyah and I. M. Singer. The index of elliptic operators I. // Ann. Math. 87:3, 484-530 (1968).

2. A.Yu. Savin, B.Yu. Sternin. On the problem of the homotopy classification of elliptic boundary value problems // Dokl. Akad. Nauk. 377:2, 165-169 (2001). [Dokl. Math. 63:2, 174-178 (2001).]

3. S. T. Melo, R. Nest, and E. Schrohe. $C^{*}$-structure and $K$-theory of Boutet de Monvel's algebra // J. Reine Angew. Math. 561, 145-175 (2003).

4. S. T. Melo, Th. Schick, and E. Schrohe. A K-theoretic proof of Boutet de Monvel's index theorem for boundary value problems // J. Reine Angew. Math. 599, 217-233 (2006).

5. A. Savin. Elliptic operators on manifolds with singularities and K-homology // K-theory. 34:1, 71-98 (2005).

6. V.E. Nazaikinskii, A.Yu. Savin, B.Yu. Sternin. Homotopy classification of elliptic operators on stratified manifolds // Izv. RAN. Ser. Matem. 71:6, 91-118 (2007). [Izv. Math. 71:6, 1167-1192 (2007).]

7. V.E. Nazaikinskii, A.Yu. Savin, B.Yu. Sternin. On the homotopy classification of elliptic operators on manifolds with corners // Dokl. Akad. Nauk. 413:1, 16-19 (2007). [ Dokl. Math. 75:2, 186-189 (2007).]

8. M. F. Atiyah and R. Bott. The index problem for manifolds with boundary // in "Bombay Colloquium on Differential Analysis", Oxford Univ. Press, Oxford, 175-186 (1964).

9. R. Melrose and F. Rochon. Index in $K$-theory for families of fibred cusp operators // K-Theory. 37:1-2, 25-104 (2006).

10. V.E. Nazaikinskii, A.Yu. Savin, B.Yu. Sternin. Noncommutative geometry and classification of elliptic operators // Sovr. Matem. Fund. Napr. 29, 131-164 (2008). [J. Math. Sci. 170:2, 229-237 (2010).]

11. V.E. Nazaikinskii, A.Yu. Savin, B.Yu. Sternin. Atiyah-Bott index on stratified manifolds // Sovr. Matem. Fund. Napr. 34, 100-108, (2009). [J. Math. Sci. 170:2, 229-237 (2010).]

12. J.-M. Lescure. Elliptic symbols, elliptic operators and Poincaré duality on conical pseudomanifolds // J. K-Theory. 4:2, 263-297 (2009).

13. B. Monthubert and V. Nistor. A topological index theorem for manifolds with corners // Compos. Math. 148:2, 640-668 (2012). 
14. A. Antonevich and A. Lebedev. Functional-Differential Equations. I. $C^{*}$-Theory. Pitman Monographs and Surveys in Pure and Applied Mathematics. 70. John Wiley \& Sons Inc, New York (1994).

15. A. B. Antonevich and A. V. Lebedev. Functional equations and functional operator equations. A $C^{*}$-algebraic approach // Trudy Sankt-Peterburg. Matem. Obsch. 6, 34-140 (1998). [Amer. Math. Soc. Transl. Ser. 2. 199, 25-116, (2000).]

16. V. E. Nazaikinskii, A. Yu. Savin, and B. Yu. Sternin. Elliptic theory and noncommutative geometry. Operator Theory: Advances and Applications. 183. Birkhäuser Verlag, Basel (2008).

17. A. Savin and B. Sternin. Elliptic theory for operators associated with diffeomorphisms of smooth manifolds // in "Pseudo-differential Operators, generalized functions and asymptotics". Operator Theory: Advances and Applications. 231, 1-26 (2013).

18. P. Baum, A. Connes, and N. Higson. Classifying space for proper actions and $K$-theory of group $C^{*}$-algebras // Contemp. Math. 167, 241-291 (1994)

19. I.Ts. Gokhberg, M.G. Krein. Systems of integral equations on the half-line with kernels depending on the difference of the arguments // Uspekhi Mat. Nauk. 13:2(80), 3-72 (1958). (in Russian).

20. M.I. Vishik, G.I. Eskin. Elliptic equations in convolution in a bounded domain and their applications // Uspekhi Matem. Nauk. 22:1, 15-76 (1965). [Russ. Math. Surv. 22:1, 13-75 (1967).]

21. G.I. Eskin. Boundary value problems for elliptic pseudodifferential equations. Nauka, Moscow (1973). [Transl. Math. Mon. 52. Amer. Math. Soc., Providence, R.I. (1981).]

22. S. Rempel and B.-W. Schulze. Parametrices and boundary symbolic calculus for elliptic boundary problems without the transmission property // Math. Nachr. 105:1, 45-149, (1982).

23. A. L. T. Paterson. Amenability. Mathematical Surveys and Monographs. 29. Amer. Math. Soc., Providence, R.I. (1988).

24. A. Antonevich, M. Belousov, and A. Lebedev. Functional differential equations. II. $C^{*}$ applications. Parts 1, 2. Pitman Monographs and Surveys in Pure and Applied Mathematics 94, 95. Longman, Harlow (1998).

25. M. Pimsner and D. Voiculescu. Exact sequences for $K$-groups and Ext-groups of certain crossproduct $C^{*}$-algebras // J. Oper. Theory. 4, 93-118 (1980).

26. B.V. Fedosov. Index theorems // Itogi Nauki Tekh. Ser. Sovrem. Probl. Mat. Fundam. Naprav. 65, 165-268 (1991). [Part. Diff. Equat. VIII. Encycl. Math. Sci. 65, 155-251 (1996).]

27. B. Blackadar. K-Theory for Operator Algebras. Mathematical Sciences Research Institute Publications. Cambridge Univ. Press, Cambridge (1998).

Anton Yurievich Savin,

RUDN University,

Moscow Miklukho-Maklaya str. 6,

117198, Moscow, Russia

Leibniz Universität Hannover,

Welfengarten 1,

D-30167 Hannover, Germany

E-mail: antonsavin@mail.ru

Boris Yurievich Sternin,

RUDN University,

Moscow Miklukho-Maklaya str. 6,

117198, Moscow, Russia

Leibniz Universität Hannover,

Welfengarten 1 ,

D-30167 Hannover, Germany

E-mail: sternin@mail.ru 